\title{
The viscosity and the thermal conductivity of normal liquid Helium 3 in the $L O C V$ frame-work
}

\author{
M. Modarres* and M. Rahmat \\ Physics Department, University of Tehran, 1439955961, Tehran, Iran.
}

\begin{abstract}
The lowest order constrained variational $(L O C V)$ method is used to evaluate the transport properties of normal liquid Helium-3 $\left({ }^{3} \mathrm{He}\right)$ within the Landau-Abrikosov-Khalatnikov (LAK) formalism. The $L O C V$ effective two-body interaction of the liquid Helium 3 is used to calculate the differential cross-section and the scattering probability, which is needed to solve the $L A K$ equations. It is shown that, the choice of effective mass has crucial role on the resulting viscosity and thermal conductivity of normal liquid ${ }^{3} \mathrm{He}$. Our $L O C V$-LAK calculations are compared with the other theoretical and experimental results.
\end{abstract}

PACS numbers: 61.20.Gy, 61.20.Ne, 67.55.-s,67.50.Dg

Keywords: $L O C V$ method,two-body effective potential, ${ }^{3} \mathrm{He}-{ }^{3} \mathrm{He}$ potential, normal liquid helium 3, scattering probability.

${ }^{*}$ Corresponding author, Email : mmodares@ut.ac.ir, Tel:+98-21-61118645, Fax:+98-21-88004781 


\section{INTRODUCTION}

In the recent years, many theoretical and experimental physicists have been interested in studying the properties of normal liquid ${ }^{3} \mathrm{He}$, since it can be applied as a working fluid in the cryogenic engineering as well as the nuclear physics. The transport properties and specially the viscosity and the thermal conductivity of Helium 3, as well as its thermodynamic properties are required for modeling, design and evaluation of thermodynamic cycles [1].

During the past decades, the precision measurements of the transport properties of ${ }^{3} \mathrm{He}$ have been carried out and also there have been many attempts to describe them theoretically. An experimental description of the transport properties of ${ }^{3} \mathrm{He}$ at low temperatures is given by Wheatley in his articles [2-4], and also a series of data for the viscosity and the thermal conductivity of ${ }^{3} \mathrm{He}$ at both the liquid and the gas phases at temperatures from $3 \mathrm{mK}$ to $500 \mathrm{~K}$ and pressures up to $20 M P a$, can be found in the references [1] and [5].

Abrikosov and Khalatnikov [6, 7] $(A K)$, based on the Landau Fermi liquid theory, have originally investigated the kinetic phenomena in the liquid ${ }^{3} \mathrm{He}$ and have formulated a theory for the transport properties of the liquid ${ }^{3} \mathrm{He}$ at low temperatures, which predicts for the viscosity and the thermal conductivity, $\eta \propto \frac{10^{-6}-10^{-5}}{T^{2}}$ poise and $\kappa \propto \frac{10^{2}-10^{3}}{T} \mathrm{erg} / \mathrm{cm} . \mathrm{s} . K$, respectively. Later, Brooker and Sykes [8] by solving certain integral equations of references $[6,7]$, corrected the AK expressions for the viscosity and the thermal conductivity at low temperatures. The liquid ${ }^{3} \mathrm{He}$ is a very exceptional system. It behaves like a normal liquid and obeys the Landau Fermi liquid theory, when the temperature is sufficiently low, i.e. $T_{c}=0.0026 K<T<0.16 K$, while it becomes superfluid below the critical temperature $T_{c}$. In this paper we deal only with the normal-liquid phase of ${ }^{3} \mathrm{He}$ and try to study its transport properties within the $L A K$ formalism by using the $L O C V$ method.

The lowest order constrained variational ( $L O C V$ ) method as well its extended one, $(E L O C V)$, have been widely and successfully used for calculating properties of homogeneous quantum fluids such as liquid ${ }^{3} \mathrm{He}$ at zero and finite temperatures [10-12], and also recently, it was used to calculate the in-medium $n n$ cross section and the transport properties of neutron matter $[13,14]$. In these calculations, a very good agreement was found between the $L O C V$ results and the more complicated approaches which go beyond the lowest order [15]. It has been also shown that the $L O C V$ formalism has very good convergence properties $[10,11,16,17]$. The advantages of $L O C V$ formalism with respect to other many-body 
techniques which go beyond the lowest order or use the simulation procedures, were fully discussed in the several of our works $[10,11,18-20]$.

So the paper is structured as follows: In the section 2, we present the uniform ${ }^{3} \mathrm{He}$ fluid Hamiltonian with a short description of the lowest order constrained variational method. The section 3 is devoted to calculation of the transport coefficients of liquid ${ }^{3} \mathrm{He}$ in the $L A K$ framework. Finally, results, discussions and conclusions are given in the section 4 .

\section{THE GENERATION OF EFFECTIVE TWO-BODY INTERACTION VIA THE LOCV FORMALISM}

The theoretical and the experimental prediction of the equation of states (EOS) of liquid ${ }^{3} \mathrm{He}$ and ${ }^{4} \mathrm{He}$ is one of the most important problems in condensed matter physics [21]. Hence, on the theoretical side, any attempt to employ such a microscopic realistic calculation is crucial. There are very high quality calculations in the HNC/FHNC framework by the Krotscheck group on liquid ${ }^{3} \mathrm{He}$ and ${ }^{4} \mathrm{He}$ at $\mathrm{T}=0$ (for example see [22]). Also, it can be found the EOS of liquid ${ }^{3} \mathrm{He}$ at the zero and the finite temperatures in the LOCV framework in the references [10] and [11], respectively. As we stated before, the two formalisms are reasonably in agreement to each other. In this section we present a short description of the LOCV formalism to obtain the EOS of liquid ${ }^{3} \mathrm{He}$ and also extract the effective two-body interaction.

The total Hamiltonian of the liquid ${ }^{3} \mathrm{He}$ is usually written as:

$$
\mathcal{H}_{{ }^{H} \mathrm{He}}=\sum_{i=1}^{N} \frac{p_{i}^{2}}{2 m}+\frac{1}{2} \sum_{i \neq j} v_{i j}
$$

where $v_{i j}$ is the interatomic potential. In this work we consider the Lennard - Jones potential [23] (other interactions such as $A z i z$ do not change the results dramatically).

The ${ }^{3} \mathrm{He}$ fluid energy in the cluster expansion technique [24], can be written as:

$$
E[f]=\frac{1}{N} \frac{<\psi|H| \psi>}{<\psi \mid \psi>}=T_{F}+E_{M B}=T_{F}+E_{2}+E_{3} \ldots
$$

In the $L O C V$ method, we use an ideal Fermi gas type wave function for the single-particle states and the variational techniques to find the wave function of the interacting system $[11,19,20]$, i.e.

$$
\psi=\mathcal{F} \phi
$$


where $\phi$ is a Slater determinant of plane waves of $\mathrm{N}$ independent nucleons,

$$
\phi=\mathcal{A} \prod_{i} \exp \left(i \vec{k}_{i} \cdot \vec{r}_{i}\right)
$$

$\mathcal{F}$ will be approximated by its Jastrow type, i.e.,

$$
\mathcal{F}=\mathcal{S} \prod_{i>j} f(i j)
$$

$\mathcal{A}$ and $\mathcal{S}$ denote the anti-symmetrization and the symmetrization operators, respectively. In general, the Jastrow correlation functions $f(i j)$ are operators. But in the case of ${ }^{3} \mathrm{He}$ fluid because of the simplicity of interatomic interaction, we assume them to depend only on the relative distance of two particles. However, the effects of the angular and the spin dependence of correlation functions, which built in, by the above Slater determinant were investigated by Modarres in the references $[25,26]$ and it is shown that there is no significant contributions from theses degrees of freedom.

In the equation (2), $T_{F}$ is simply the Fermi gas kinetic energy, and is defined by, $(\Omega$ stands for the volume),

$$
T_{F}=(\Omega \rho)^{-1} \sum_{k, \sigma} \frac{k^{2}}{2 m} \theta\left(k_{F}-k\right) .
$$

The fluid density $\rho$ will fix the Fermi-momentum $k_{F}\left(=\left(3 \pi^{2} \rho\right)^{1 / 3}\right)$ and the $E_{2}$ term i.e. the two-body energy is defined as:

$$
E_{2}=\frac{1}{N} \sum_{i<j}\langle i j|\mathcal{V}(12)| i j\rangle_{a}
$$

where the " effective two-body interaction " $\mathcal{V}(12)$, is given by the following equation:

$$
\mathcal{V}(12)=-\frac{\hbar^{2}}{2 m}\left[f(12),\left[\nabla_{12}^{2}, f(12)\right]\right]+f(12) v(12) f(12)
$$

and the two-body anti-symmetrized matrix element $\langle i j|\mathcal{V}| i j\rangle_{a}$ are taken with respect to the single-particle functions composing $\phi$, i.e. the plane-waves.

In the $L O C V$ formalism, the $E_{M B}$ term, is approximated by $E_{2}$, and one hopes that the normalization constraint, $\langle\psi \mid \psi\rangle=1$, makes the cluster expansion converges very rapidly and bring the many-body effect into the $E_{2}$ term (as we discussed earlier and in the reference $[10])$. 
For the ${ }^{3} \mathrm{He}$ fluid, because of the choice of correlation functions, the two-body effective interaction in the equation (8) is reduced to the following equation (note that in contrast to the nucleonic matter case, there is no approximation $)\left(r=\left|\mathbf{r}_{1}-\mathbf{r}_{\mathbf{2}}\right|\right)$ :

$$
\mathcal{V}(r)=\frac{\hbar^{2}}{m}(\nabla f(r))^{2}+f^{2}(r) v(r) .
$$

where $v(r)$ is the ${ }^{3} \mathrm{He}-{ }^{3} \mathrm{He}$ interaction. Now, by using plane waves as the single-particle states, equation (7) simply reduces to:

$$
E_{2}=\frac{1}{2} \rho \int \mathrm{g}_{F}(r) \mathcal{V}(r) d \mathbf{r}
$$

where

$$
\mathrm{g}_{F}(r)=\left[1-\frac{1}{2} l^{2}\left(k_{F} r\right)\right]
$$

and the Pauli function is defined as

$$
l(x)=\frac{3 J_{1}(x)}{x}
$$

In the above equation, $J_{l}(x)$ are the familiar spherical Bessel functions. On the other hand the normalization constraint can also be written as follows:

$$
\langle\psi \mid \psi\rangle=\rho \int \mathrm{g}_{F}(r) f^{2}(12) d \mathbf{r}
$$

Note that $\xi=[\langle\psi \mid \psi\rangle-1]$ plays the role of the smallness parameter in the cluster expansion. The above constraint introduces another parameter in our formalism, i.e. the Lagrange multiplier $\lambda$. So at each density we minimize the functional $\mathcal{L}=\left\{E_{2}+\lambda[\langle\psi \mid \psi\rangle\}[f]\right.$ with respect to $f(r)$ by solving the Euler-Lagrange equation $\left(f^{\prime}=\frac{\partial f(r)}{\partial r}\right)$, i.e.

$$
\frac{\partial \mathcal{L}}{\partial f(r)}-\frac{\partial}{\partial r} \frac{\partial \mathcal{L}}{\partial f^{\prime}(r)}=0
$$

and we choose $\lambda$ such that the above normalization constraint is satisfied. The EulerLagrange equation has the following explicit form:

$$
g^{\prime \prime}(r)-\left[\frac{A^{\prime \prime}(r)}{A(r)}+\frac{m}{\hbar^{2}}(v(r)-\lambda)\right] g(r)=0,
$$

where $g(r)=A(r) f(r)$ and $A^{2}(r)=r^{2} \mathrm{~g}_{F}(r)$. For given $\lambda$, the above differential equation is solved such that, the correlation function heals to the Pauli function $f_{p}(r)=\mathrm{g}_{F}(r)^{-1 / 2}$ and finally $\lambda$ is fixed by the constraint. The calculated correlation functions, $f(r)$, at each 
density, which are the out come of the equations (13) to (15), are inserted into the equation (9) to generate the effective two-body potential for the liquid helium 3.

So, in this way, we can get the binding energy per particle versus the density $(E O S)$ for liquid ${ }^{3} \mathrm{He}$ in the $L O C V$ formalism (as it has been plotted in reference [10]) and then, by

using the formula: $P=\rho^{2} \frac{\partial E}{\partial \rho}$, we can obtain the pressure corresponding to the density for liquid ${ }^{3} \mathrm{He}$, as it has been plotted in the figure $4 \mathrm{a}$ of the reference [11]. In this paper, the figure $4 \mathrm{~b}$ shows the experimental data for the pressure of liquid ${ }^{3} \mathrm{He}$, that it confirms the existence of a liquid phase for the region $T<2.5 \mathrm{~K}$ and densities $0.0164<\rho<0.0174 A^{\mathrm{\circ}^{-3}}$ and $P<300 a t m$.

\section{THE TRANSPORT PROPERTIES OF LIQUID ${ }^{3} \mathrm{He}$}

Based on the Landau Fermi liquid model [27], Abrikosov and Khalatnikov formulated a theory for the transport properties of the interacting Fermi liquids at low temperature. Their results for the viscosity reads as follows:

$$
\eta_{A K}=\frac{1}{5} \rho m^{*} \nu_{F}^{2} \tau \frac{2}{\pi^{2}\left(1-\lambda_{\eta}\right)},
$$

where $\nu_{F}=k_{F} / m^{*}$ is the Fermi velocity, and $m^{*}$ and $\tau$ denote the quasi-particle effective mass and the lifetime, respectively. The quantity $\lambda_{\eta}$ in the equation (16) is defined as,

$$
\lambda_{\eta}=\frac{\left\langle W\left[1-3 \sin ^{4}(\theta / 2) \sin ^{2}(\phi)\right]\right\rangle}{\langle W\rangle},
$$

and the lifetime of quasi-particle, $\tau$, can be written in terms of the angle-averaged scattering probability, $\langle W\rangle$, as

$$
\tau T^{2}=\frac{8 \pi^{4}}{m^{*^{3}}} \frac{1}{\langle W\rangle}
$$

with

$$
\langle W\rangle=\int \frac{d \Omega}{2 \pi} \frac{W(\theta, \phi)}{\cos (\theta / 2)},
$$

where $d \Omega=\sin \theta d \theta d \phi$ with $0 \leq \theta \leq \pi$ and since the particles are indistinguishable then $0 \leq$ $\phi \leq \pi$. In above equation, these assumptions have been made that, at low temperature, $T$, the collisions are restricted to the vicinity of Fermi surface and therefore for any given density $\rho, \mathrm{W}$ depends only on the angular variables $\theta$ and $\phi$, and the magnitude of all quasi-particles momenta being equal to the Fermi momentum $k_{F}$. 
Brooker and Sykes [8] have obtained the exact solution of the equation (16) which has been derived in the reference [6], as follows,

$$
\eta=\eta_{A K} C\left(\lambda_{\eta}\right)
$$

where

$$
C\left(\lambda_{\eta}\right)=\frac{1-\lambda_{\eta}}{4} \sum_{k=0}^{\infty} \frac{4 k+3}{(k+1)(2 k+1)\left[(k+1)(2 k+1)-\lambda_{\eta}\right]} .
$$

With the same procedure, the thermal conductivity, $\kappa$, can also be obtained as,

$$
\kappa=\kappa_{A K} H\left(\lambda_{\kappa}\right)
$$

where

$$
\kappa_{A K}=\frac{1}{T} \frac{8}{3} \frac{k_{F}^{3}}{m^{*^{3}}} \frac{2 \pi^{2}}{\langle W\rangle\left(3-\lambda_{\kappa}\right)}
$$

with the correction factor, $H\left(\lambda_{\kappa}\right)$,

$$
H\left(\lambda_{\kappa}\right)=\frac{3-\lambda_{\kappa}}{4} \sum_{k=0}^{\infty} \frac{4 k+5}{(k+1)(2 k+3)\left[(k+1)(2 k+3)-\lambda_{\kappa}\right]},
$$

in which

$$
\lambda_{\kappa}=\frac{\langle W[1+2 \cos (\theta)]\rangle}{\langle W\rangle} .
$$

The in-medium effects in two-body collisions can be incorporated through two central quantities: (i) the two-body scattering rates and, (ii) the quasi-particle effective mass. In the case (i), as it can be seen from the above equations, the key element in the determination of the viscosity and the thermal conductivity is the in-medium two-body scattering probability, W. The scattering probability is usually obtained from the Landau parameters of the Fermi liquids [27]. Indeed in this approach, the interaction among the quasi-particles is parameterized by means of a set of constants known as the Landau parameters. The Landau coefficients are the phenomenological parameters to be determined from the experiment. But in this work, we intend to obtain the scattering probability by using the $L O C V$ effective two-body interactions, i.e. the equation (9), as it was applied in the case of pure neutron matter $(P N M)$ in our previous works $[13,14]$. So, with this approach, we can include the many-body effects of the liquid medium in the scattering amplitude. 
In our approach, knowing the $L O C V$ effective two-body interactions, the in-medium scattering probability can be obtained from the Fermi golden rule (see the appendix A). The corresponding cross section at momentum transfer $q$ reads as,

$$
\frac{d \sigma}{d \Omega}=\frac{m^{*^{2}}}{16 \pi^{2}}|\hat{\nu}(q)|^{2}
$$

where $\hat{\nu}(q)$ is the Fourier transform of the $L O C V$ effective two-body interactions i.e. $\mathcal{V}(r)$. Then the scattering probability, $W(\theta, \phi)$, can be easily extracted from the cross section:

$$
W(\theta, \phi)=\frac{16 \pi^{2}}{m^{*^{2}}}\left(\frac{d \sigma}{d \Omega}\right) .
$$

In the case (ii), as it was explained before, $m^{*}$ as the quasi-particle effective mass can also play the role of the in-medium effect. Because of the interactions between the quasiparticles in ${ }^{3} \mathrm{He}$, instead of the bare mass, the effective mass is used. Where it is defined by $\nu_{F}=k_{F} / m^{*}$ and it can be obtained from the quasi-particle energies computed in the Hartree - Fock approximation ( $e(k)$ is the single particle energies):

$$
m^{*}(k)=\frac{\hbar^{2} k}{\left(\frac{d e(k)}{d k}\right)} .
$$

However, in the most cases, $m^{*}$ is determined from the Landau parameters [27]:

$$
\frac{m^{*}}{m}=1+\frac{F_{1}^{s}}{3}
$$

and it is usually extracted from the experimental specific heat data of ${ }^{3} \mathrm{He}$ at low temperatures. It is observed that $m^{*} / m$ varies strongly with pressure.

Due to the high density and the strong inter-particle interactions in the liquid ${ }^{3} \mathrm{He}$, the determination of the effective mass has always been a challenge in physics, nevertheless there were some serious attempts to evaluate the quasi-particle effective mass by using the microscopic methods.

Brueckner and Gammel (BG) [29], using the Green's function method obtained a value of $m^{*} / m=1.84$, which is quite far off the experimental data. Subsequently, an improvement over the $B G$ theory was made by Ostagaard [30] who used $m^{*} / m$ as an adjustable parameter in his theory, to obtain the best value for the average binding energy per particle [31] of the helium 3 liquid. By this choice, he obtained a binding energy of $-2.0 K$ corresponding to a value of $m^{*} / m=2.5$. Tan and Feenberg [32], by using the method of correlated basis 
functions have found $m^{*} / m=2.28$. In the framework of correlated basis perturbation theory (CBPT), Fantoni and Pandharipande [33] have predicted the effective mass to have values as low as $0.76 \mathrm{~m}$. Tripathy and Mishra [31] in a self-consistent method have obtained the value of $m^{*} / m$ to be 2.99. In their paper, a more discussion about the effective mass of ${ }^{3} H e$ can be found. They pointed out that, it seems that the theoretical determination of the effective mass of a helium atom in the liquid phase has not yielded a satisfactory result so far.

In this paper, we intend to use the effective mass of ${ }^{3} \mathrm{He}$ for the calculations of the transport properties of liquid helium 3, by performing the two approaches: First, we derive the effective mass from the single particle energies computed in the $L O C V$ framework $(S P E-L O C V)$. In this direction The single particle energy is defined as follows:

$$
e\left(k_{i}\right)=\frac{\hbar^{2} k_{i}^{2}}{2 m}+U\left(k_{i}, \rho\right)
$$

where the density dependent single particle potential $U(k, \rho)$ is determined by the equation (note that $\rho$ is a function of $k_{F}$ ):

$$
U\left(k_{i}, \rho\right)=\sum_{j}\langle i j|\mathcal{V}(12)| i j\rangle_{a} .
$$

After doing some algebra, one find the following equation for the single particle potential in the lowest order level:

$$
U\left(k_{i}, \rho\right)=\rho \int\left[1-\frac{1}{2} l\left(k_{F} r\right) J_{0}\left(k_{i} r\right)\right] \mathcal{V}(r, \rho) d \mathbf{r} .
$$

Then, the density dependent effective mass can be determined by the variation of the single particle energy with respect to the momentum $k$, i.e. the equation (28). After the evaluation of effective mass, one can calculate the transport coefficients of liquid ${ }^{3} \mathrm{He}$ (as we have done for $P N M)[13,14]$. But, in the second approach, we consider the effective mass as an adjustable parameter to obtain the best value for transport coefficients of liquid ${ }^{3} \mathrm{He}$ with respect to the experimental data.

\section{RESULTS, DISCUSSIONS AND CONCLUSIONS}

The $L O C V$ effective two body interactions for the liquid helium 3 are given in the figure

1 for the three different densities (pressures) (see the equation (9)). As one should expect, as 
the densities (pressures) increase the repulsion parts of effective interactions become more stronger. Similarly, in the Figure 2 the difference between the $L O C V$ single particle energies at given momentums $\mathrm{k}$ and that of Fermi momentums $k_{F}$ are plotted versus the ration $k / k_{F}$. Obviously, for given density or pressure, the single particle energies are less than the one at the Fermi surface. On the other hand as we increase the densities or the pressures, their differences become larger. The results of reference [33] which is base on the correlated-basis perturbation theory, $C B P T$, is also given for comparison.

The table 1 shows the results of calculation of the effective mass of the liquid ${ }^{3} \mathrm{He}$ at the Fermi momentum $k_{F}$, using the calculated lowest order single particle energies in the $L O C V$ method, i.e. the equations (28) and (30), at the different pressures. As it can be seen from this table, at $P=50 \mathrm{~atm}$, we have obtained $m^{*} / m=0.74$, which is in a very good agreement with the results of Fantoni and Pandharipande [33]. They have obtained $m^{*} / m=0.76$ in the first-order CBPT. The table 1 shows, $m^{*} / m$ decreases with the density, but it is a well known result of ${ }^{3} \mathrm{He}$ phenomenology that $m^{*} / m$ increases with density (for example see the reference [34]). The same problem has been reported in the reference[34] in the frame work of the first-order CBPT .

Our effective mass results, $m^{*}\left(k_{F}\right)$, are much smaller than the experimental values, which is obtained from the experimental specific heat data. It is well known that $m^{*} / m$ should be bigger than one for the liquid ${ }^{3} \mathrm{He}[35,36]$. Also, as it will be shown in the tables 2 and 3 , the transport coefficients of liquid ${ }^{3} \mathrm{He}$, using the $m^{*}$ of $S P E-L O C V$ are not in agreement with its experimental values and the results of reference [27].

These discrepancies can be due to the properties of liquid ${ }^{3} \mathrm{He}$ which is a highly dense system with the strong inter-particle interaction. So, by taking into the account only the two-body correlations and energy in the cluster expansion, it may not be adequate to predict good results, specially at high densities, and the inclusion of three body and many body effects may play some roles in the calculations. It can be seen in the reference [10] that inclusion of three body cluster energy and specially the role of our normalization constraint on the higher cluster terms in the liquid ${ }^{3} \mathrm{He}$ (the $E L O C V$ approaches) improves the results. But n contrast, in the case of $P N M[13,14]$, we obtained very good results only by inclusion of two body effects, but since in the liquid ${ }^{3} \mathrm{He}$, atoms are approximately three times more compressed than the nucleons in the nucleonic matter, we did not get very good results for the liquid helium 3. So in our future works, as it was stated before, we should take into 
account the high cluster effects in the ${ }^{3} \mathrm{He}$ liquid calculations and investigate the possible sources of this discrepancies. However, based on $C B P T$ results this may still be an open problem.

In order to obtain the closest value of the transport coefficients of liquid ${ }^{3} \mathrm{He}$ to experimental data, we consider the effective mass as an adjustable parameter. The values of adjustable $m^{*}$ and the corresponding transport coefficients are given in the tables 2 and 3 . As it can be seen, we have found the value of $m^{*} / m$ to be about 2 , that is comparable with the values of $m^{*} / m=2.2$ and $m^{*} / m=2.9$ according to Alvesalo et al [35] and Mota et al [36], by measuring the specific heat of liquid ${ }^{3} \mathrm{He}$, respectively. Therefore, by choosing the effective mass as an adjustable parameter, we can obtain reasonable results for the viscosity and the thermal conductivity of liquid ${ }^{3} \mathrm{He}$ which are in a good agreement with the other theoretical and experimental results.

In conclusion, the transport properties of normal liquid Helium-3 $\left({ }^{3} \mathrm{He}\right)$ were obtained within the Landau - Abrikosov - Khalatnikov ( $L A K)$ formalism and the lowest order constrained variational $(L O C V)$ method. The $L O C V$ effective two-body interaction of the liquid Helium 3 was used to calculate the differential cross-section and the scattering probability, which is needed to solve the $L A K$ equations. It was shown that, the choice of effective mass has crucial role on the resulting viscosity and thermal conductivity of the normal liquid ${ }^{3} \mathrm{He}$. Our $L O C V-L A K$ calculations were compared with the other theoretical and experimental results. We hope in future, by including the three-body cluster free energy and taking into the account the temperature, we could understand the discrepancies we faced i.e. the decreasing (rather raising) of effective mass of liquid helium 3 against the density, in the present work.

\section{Acknowledgments}

The authors would like to acknowledge the Research Council of University of Tehran and the Iran National Science Foundation $(I N S F)$ for the grants provided for them. 


\section{APPENDIX A: THE CALCULATION OF THE CROSS SECTION}

At low temperatures, the density of thermally excited ${ }^{3} \mathrm{He}$ quasi-particles is low, and it is only necessary to consider collisions of two quasi-particles at a time [27]. In this process, two ${ }^{3} \mathrm{He}$ quasi-particles with the initial momentums $\mathbf{k}_{1}$ and $\mathbf{k}_{2}$ are scattered to final state of momenta $\mathbf{k}_{1}^{\prime}$ and $\mathbf{k}_{2}^{\prime}$. The total energy of initial state can be written in terms of the center of mass and relative momenta, $\mathbf{K}=\mathbf{k}_{1}+\mathbf{k}_{2}$ and $\mathbf{k}=\left(\mathbf{k}_{1}-\mathbf{k}_{2}\right) / 2$, as

$$
E=\frac{K^{2}}{2 M}+\frac{k^{2}}{2 \mu}=\varepsilon+\varepsilon_{r e l}
$$

where $M=2 m^{*}$ and $\mu=m^{*} / 2$ is reduced mass.

In the center of mass $(\mathrm{CM})$ frame, the reference frame, in which the center of mass of the system is at rest i.e. $\mathbf{K}=0$, we will have $E=E_{C M}=\varepsilon_{r e l}$. The scattering process are often considered in the CM frame.

At low temperature limit, the collisions only occur near the Fermi surface, this means that:

$$
\left|\mathbf{k}_{1}\right|=\left|\mathbf{k}_{2}\right|=\left|\mathbf{k}_{1}^{\prime}\right|=\left|\mathbf{k}_{2}^{\prime}\right|=k_{F},
$$

and for an elastic scattering: $|\mathbf{k}|=\left|\mathbf{k}^{\prime}\right|$, where $k^{\prime}$ is the relative momentum after the scattering. So we can write the scattering energy in the CM frame as,

$$
E_{C M}=\frac{k_{F}^{2}}{2 m^{*}}(1-\cos \theta),
$$

where, $\theta$ is the angle between the incoming quasi-particle momenta $\mathbf{k}_{1}$ and $\mathbf{k}_{2}$, and the center of mass scattering and the angle $\theta_{C M}=\phi$, is the angle between the initial and final relative momenta, $\mathbf{k}$ and $\mathbf{k}^{\prime} ; \cos \phi=\frac{\mathbf{k} \cdot \mathbf{k}^{\prime}}{|\mathbf{k}|\left|\mathbf{k}^{\prime}\right|}$.

By working in the CM frame, we have effectively one-particle problem, and this particle can make a transition from an initial state, $|i\rangle$, to the final state, $|f\rangle$. The transition rate, following the Fermi's Golden Rule is given by [37]:

$$
\mathcal{R}_{i \rightarrow f}=2 \pi \int \frac{V d \mathbf{k}^{\prime}}{(2 \pi)^{3}}\left|\mathcal{M}_{f i}\right|^{2} \delta\left(\frac{k^{\prime^{2}}}{2 \mu}-\frac{k^{2}}{2 \mu}\right)
$$

The delta function expresses energy conversation. The matrix element $\mathcal{M}_{f i}$, is the transition amplitude:

$$
\mathcal{M}_{f i}=\left\langle f\left|\mathcal{V}_{e f f}\right| i\right\rangle_{a}=\left\langle\mathbf{k}^{\prime}, S M^{\prime}\left|\mathcal{V}_{e f f}\right| \mathbf{k}, S M\right\rangle_{a}
$$


By carrying out the delta function integration, we get following equation:

$$
\mathcal{R}_{i \rightarrow f}=\frac{1}{4 \pi^{2} V} \int d \Omega k^{\prime} \mu\left|\mathcal{M}_{f i}\right|^{2}
$$

In the scattering problems, the cross section i.e. the number of transitions for unit flux, is usually measured. Therefore we have:

$$
d \sigma=\frac{1}{4 \pi^{2}} \frac{1}{\left|\nu_{r e l}\right|} d \Omega k^{\prime} \mu\left|\mathcal{M}_{f i}\right|^{2} .
$$

Since in the CM frame, two incident particles are moving toward each other with equal and opposite momenta, so their relative velocity, $\nu_{r e l}$, is: $\frac{k}{\mu}$.

Then we can rewrite the differential cross section as:

$$
\frac{d \sigma}{d \Omega}=\frac{\mu^{2}}{4 \pi^{2}}\left|\mathcal{M}_{f i}\right|^{2}
$$

where, for identical particles: $\mu=\frac{m^{*}}{2}$. According to the equation (A5) and after doing some algebra, we get the following equation for the differential cross section:

$$
\frac{d \sigma}{d \Omega}=\frac{m^{*^{2}}}{16 \pi^{2}}|\hat{\nu}(q)|^{2}
$$

$\hat{\nu}(q)$ is the Fourier transform of the effective two-body matrix element of the equation (9) and $\mathbf{q}=\mathbf{k}-\mathbf{k}^{\prime}$ is the momentum transfer. Therefore the scattering probability, $W(\theta, \phi)$, can be extracted from the cross section:

$$
W(\theta, \phi)=\frac{16 \pi^{2}}{m^{*^{2}}}\left(\frac{d \sigma}{d \Omega}\right) .
$$

[1] Y. Huang, Q. Yu, Q. Chen, R. Wang, Cryogenics 52 (2012) 538.

[2] J.C. Wheatley, in Progress in Low Temperature Physics Vol. VI (North-Holland Publishing, Amsterdam, (1970) 77 .

[3] J.C. Wheatley, Phys.Rev., 165 (1968) 304.

[4] J.C. Wheatley, Rev.Mod.Phys., 47 (1975) 415.

[5] Y. Huang, L. Fang, X. Wang, R. Wang, L.Xu , AIP Conf.Proc., 1434 (2012) 1849.

[6] A.A. Abrikosov and I.M. Khalatnikov, Soviet.Phys.JETP, 55 (1957) 887.

[7] A.A. Abrikosov and I.M. Khalatnikov, Rep.Prog.Phys., 22 (1959) 329. 
[8] G. Brooker, and J. Sykes, Phys.Rev.Lett., 21 (1968) 279.

[9] L.D. Landau, Zh .Eksp.Teor.Fiz., 30 (1956) 1058.

[10] M. Modarres, H.R. Moshfegh, K. Fallahi, Eur.Phys.J.B, 36 (2003) 485.

[11] M. Modarres, H.R. Moshfegh, Physica.A, 388 (2009) 3297.

[12] A. Rajabi, M. Modarres, J.Low.Temp.Phys., 162 (2011) 182.

[13] M. Modarres, M. Rahmat, Nucl.Phys.A, 903 (2013) 40.

[14] M. Modarres, M. Rahmat, Nucl.Phys.A, 921 (2014) 19.

[15] G.H. Bordbar, M. Modarres, Phys.Rev.C, 57 (1998) 714.

[16] M. Modarres, J.M. Irvine, J.Phys.G:Nucl.Phys., 5 (1979) 7.

[17] M. Modarres, A. Rajabi, H.R. Moshfegh,Phys.Rev.C, 76 (2007) 064311.

[18] M. Modarres, Euro.Phys.Lett., 3 (1987) 1083.

[19] J.C. Owen, R.F. Bishop, J.M. Irvine, Nucl.Phys.A, 277 (1977) 45.

[20] M. Modarres, J.M. Irvine, J.Phys.G, 5 (1979) 511.

[21] C.A. Croxton, Progress in Liquid Physics, Wiley Co., London (1977).

[22] E. Krotscheck, M. Saarela, Phys. Rep. 232 (1993) 1.

[23] J. de Boer, A. Michels, Physica., 6 (1939) 409.

[24] J. Clark, Prog.Part.Nucl.Phys., 2 (1979) 89.

[25] M. Modarres, Mod.Phys.Lett.B, 19 (2005) 1793.

[26] M. Modarres, J.Low.Temp.Phys., 139 ( 2005) 387.

[27] G. Baym and C. Pethick, Landau Fermi-Liquid Theory (John Wiley \& Sons, New York, 1991).

[28] O. Benhar and M. Valli, Phys.Rev.Lett., 99 (2007) 232501.

[29] K.A. Brukner, J.A. Gammel, Phys.Rev., 109 (1985) 1040; 121 (1961) 1863.

[30] E. Ostgaard, Phys.Rev., 176 (1968) 351.

[31] D.N. Tripathy, L.k. mishra, Physica B, 162 (19900) 300.

[32] Hing- Tat Tan, E.Feenberg, Phys.Rev., 176 (1968) 370.

[33] S. Fantoni, V.R. Pandharipande, K.E. Schmidt, Phys.Rev.Lett., 48 (1982) 878.

[34] M. Kollar and D. Vollhardt, Phys.Rev.B, 61 (2000) 15347.

[35] T.A. Alvesalo, T. Haarasoja, M. T, Manninen, and A.T. Soinne, Phys.Rev.Lett., 44 (1980) 1076.

[36] A.C. Mota, R.P. Platzeck, R. Rapp, and J.C. Wheatley, Phys.Rev., 177 (1969) 266.

[37] S. Gasiorowicz, Quantum Physics (John Wiley \& Sons, New York, 1996). 
FIG. 1: The $L O C V$ effective two-body potential for normal liquid helium 3 at three different pressure and density.

FIG. 2: The $L O C V$ single particle energy with respect to that of Fermi momentum at three different pressure and density. The diamonds are from reference [33]. 
TABLE I: The effective mass ratio of liquid ${ }^{3} \mathrm{He}$ at Fermi surface obtained by using the $S P E-$ $L O C V$ method for three different pressures.

\begin{tabular}{|c|c|c|}
\hline pressure (atom) & $m^{*} / m$ & $\rho\left(A^{\circ}\right)$ \\
\hline 0.28 & 1.06 & 0.0117 \\
27 & 0.833 & 0.0153 \\
50 & 0.74 & 0.0166 \\
\hline
\end{tabular}

TABLE II: The comparison of theoretical and experimental values of the transport coefficients of liquid ${ }^{3} \mathrm{He}$ in the extreme low-temperature limit at pressure $\mathrm{P}=0.28 \mathrm{~atm}\left(\rho=0.0117 A^{\circ-3}\right)$

\begin{tabular}{|c|c|c|c|c|}
\hline method & $m^{*} / m$ & $\begin{array}{c}\kappa T \\
(\mathrm{erg} / \mathrm{cmsec})\end{array}$ & $\begin{array}{c}\eta T^{2} \\
\left(\mu P K^{2}\right)\end{array}$ & Ref. \\
\hline Experiment & & 35 & 1.8 & {$[2],[3],[4]$} \\
s- and p-wave approximation & 3 & 39 & 2.2 & {$[23]$} \\
Forward-scattering approximation & 3 & 54 & 2.8 & {$[23]$} \\
SPE - LOCV & 1.06 & 66.61 & 2.449 & \\
Best Fit - LOCV & 2.15 & 39.35 & 1.44 & \\
\hline
\end{tabular}

TABLE III: The same as table 2 but for pressure $\mathrm{P}=27 \mathrm{~atm}\left(\rho=0.0153 A^{\circ-3}\right)$

\begin{tabular}{|c|c|c|c|c|}
\hline method & $m^{*} / m$ & $\begin{array}{c}\kappa T \\
(\text { erg } / \text { cmsec })\end{array}$ & $\begin{array}{c}\eta T^{2} \\
\left(\mu P K^{2}\right)\end{array}$ & Ref. \\
\hline Experiment & & 12.9 & 1.06 & {$[2],[3],[4]$} \\
s- and p-wave approximation & 6 & 8.6 & 0.54 & {$[23]$} \\
Forward-scattering approximation & 6 & 13 & 0.76 & {$[23]$} \\
SPE - LOCV & 0.83 & 179.08 & 5.042 & \\
Best Fit - LOCV & 2.6 & 18.77 & 0.528 & \\
\hline
\end{tabular}




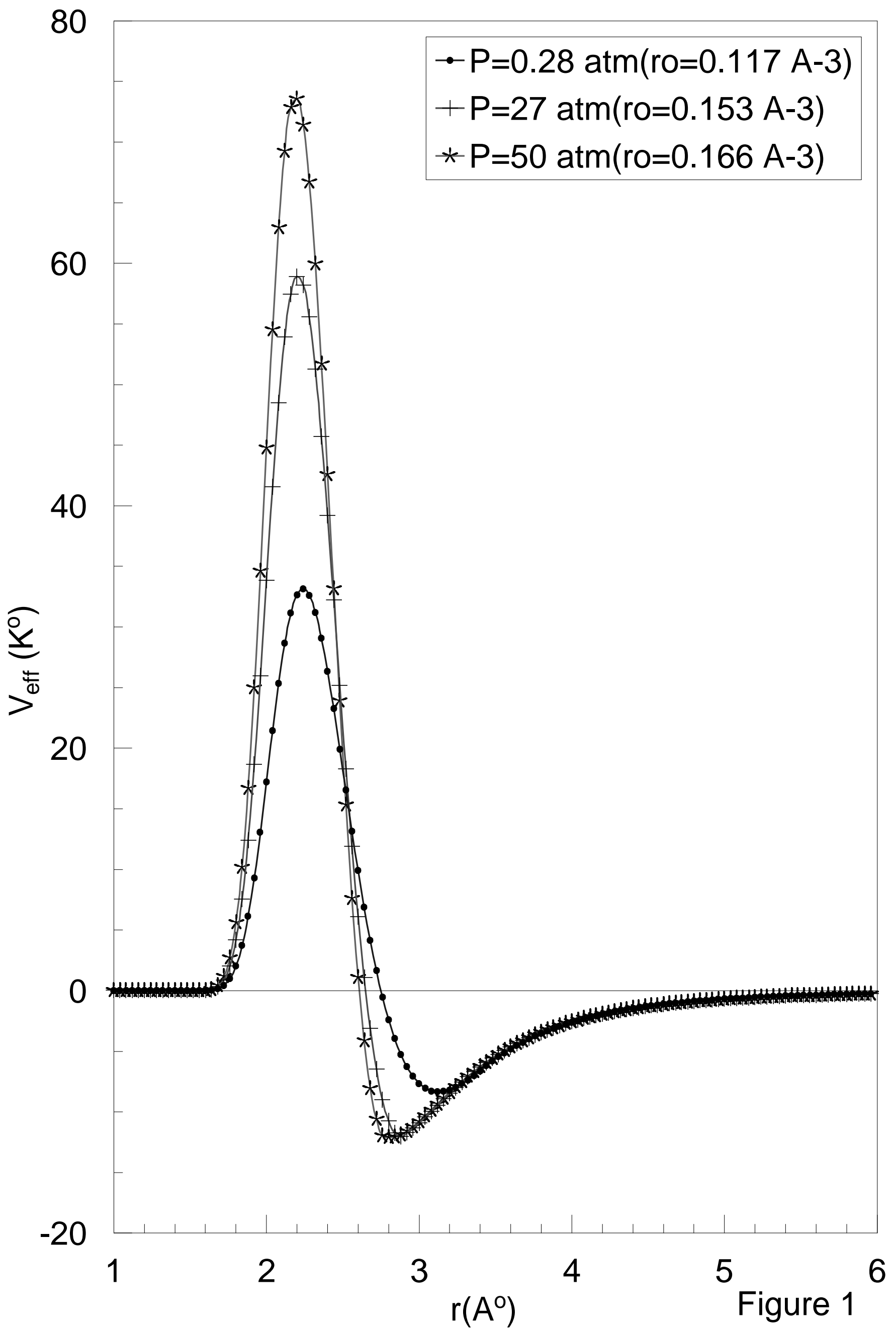




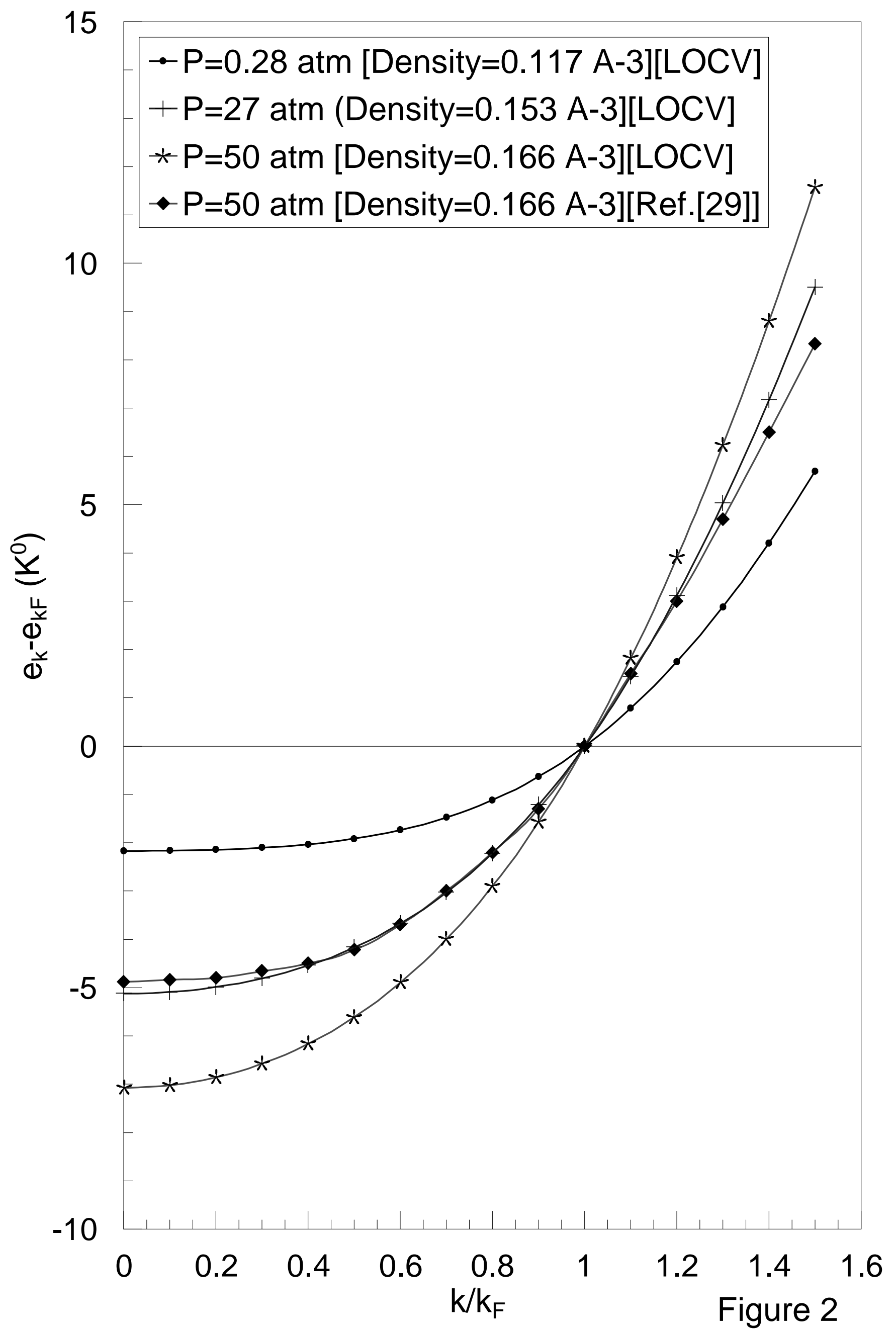

